\title{
Real-Time Ethernet Networks for Motion Control
}

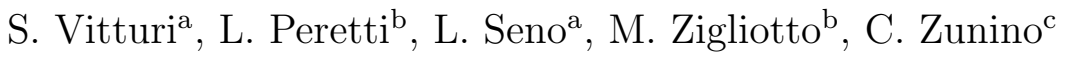 \\ ${ }^{a}$ Italian National Council of Research, IEIIT-CNR Padova and Department of \\ Information Engineering, University of Padova, Italy \\ ${ }^{b}$ Department of Technique and Management of Industrial Systems, University of Padova, \\ Vicenza, Italy \\ ${ }^{c}$ Italian National Council of Research, IEIIT-CNR Torino, Italy
}

\begin{abstract}
Communication networks have been traditionally employed in motion control applications, especially within factory automation systems. While in the past they were merely used to exchange non time critical data (e.g. parameters and configuration data) nowadays they allow for much more powerful performance. In particular, the recently introduced Real Time Ethernet (RTE) networks, have been explicitly designed in order to cope with very tight time constraints in terms of both determinism and real-time. In this paper we focus on two popular RTE networks, namely Ethernet POWERLINK and EtherCAT, and provide an example of their employment for a coordinated motion control application. In particular, we consider the tracking of a circular trajectory by the coordinated motion of two independent axes where the velocity set-points are transmitted to the electrical drives implementing the axis control by means of the RTE networks. After providing some essential features of the two networks, we describe the configurations adopted for the coordinated motion control application. Then we check the effectiveness of the solution proposed by means of numerical simulations which take into consideration possible error scenarios deriving from the adoption of the communication networks such as transmission errors, communication delays and cable breaks.
\end{abstract}

Keywords: Real-Time Ethernet, Coordinated Motion Control, Ethernet POWERLINK, EtherCAT 


\section{Introduction}

Electrical drives have been widely employed in various applications of (hierarchical) factory automation systems [1] since long time and, particularly, in motion control systems. In this context, two recently issued standardization documents have a relevant importance, namely the International Standard IEC 61800 - 7 [2], which defines a generic interface between automation systems and Power Drive Systems (PDSs), and the IEC Technical Report TR 62390 [3], which is generally concerned with automation devices profiles and hence with electrical drives as well.

According to the aforementioned documents, an effective way of integrating electrical drives in factory automation systems, confirmed by several practical applications, is represented by the use of communication networks [4]. Actually, various types of networks have been employed ranging from simple proprietary connections (e.g. dedicated serial links) up to structured communication systems defined by International Standards, depending on the specific requirements of the applications. In this scenario, particularly in the last decade, fieldbuses [5], [6] have represented a natural and effective choice, as witnessed by the impressive growth of their installations. This has suggested many suppliers of electrical drives to directly equip their devices with interfaces compliant with the most popular fieldbuses such as, for example, Profibus DP [7], DeviceNet [8], ControlNet [9], CANopen [10] to mention some. As a further interesting feature, drive profiles [11], [12] have been proposed by some fieldbus organizations in order to achieve interoperability between devices of different manufacturers.

The connection to a network allows, in principle, electrical drives to exchange data with all the nodes connected to the same communication system; in particular, they communicate with one or more "controllers" which are the devices that actually execute the automation tasks. Typical data exchanged are parameters, set-points, alarms, process values, etc. The performance of fieldbuses, however, may result unsatisfactory for some relevant applications involving electrical drives like, for example, (coordinated) motion control (with the remarkable exception represented by Sercos [13], which is a fieldbus specifically conceived to achieve drive synchronization). Indeed, the limited transmission speeds of some fieldbuses, along with a (possibly) low protocol efficiency, may prevent the timed transmission of the set-points to the drives control loops, as pointed out in [11]. Further, supposing that a controller queries periodically two or more electrical drives, connected to a 
fieldbus, in order to realize a coordinated motion control application, then the jitter that may affect the cycle time of the network [14] inevitably reflects on the polling operations, possibly causing undesired behaviors.

The introduction of Real-Time Ethernet (RTE) networks [15] allows, at least in principle, to overcome most of the above limitations. RTE networks are high speed communication systems (100/1000 Mbits/s) based on the wellknown original Ethernet specification [16] on purpose designed for industrial applications in order to guarantee very short transmission times as well as tight determinism for periodic traffic and hard real-time operation for acyclic data delivery [17]. These networks are often referred as isochronous, although such a term is used in various other contexts as well, even with different meanings ${ }^{1}$.

Several RTE network components are already commercially available from different manufacturers, and most of the networks they comply with are encompassed by both the IEC 61158 [18] and IEC61784-2 [19] International Standards.

In this paper we focus on two popular RTE networks defined by IEC 61158, namely Ethernet POWERLINK (EPL) [20] and EtherCAT [21], and investigate the possibility of using them for coordinated motion control applications. The choice of the two specific networks, among those available, has been suggested by their popularity as well as by their noticeably different technical features which, as it will be shown in the next sections, make them representatives of two different families of networks.

We start from the definition of a set of performance indicators for both the addressed RTE networks (some of them are specified in [19], additional ones will be defined in the paper). Then we consider, as a test bench, a coordinated two-axis motion control application where the velocity set-points are delivered by a controller to two electrical drives, connected by means of both the RTE networks. In particular, we firstly focus on the possible network configurations as well as on the polling schemes of their nodes. Subsequently we calculate, for both the networks, the most relevant parameters and the performance indicators. Finally, the behavior of the coordinated motion control application is investigated, under different operational conditions, by means of some tracking error indexes determined via numerical simulations. It is

\footnotetext{
${ }^{1}$ see, for example, the definitions provided in http://en.wikipedia.org/wiki/Isochronous and http://www.webopedia.com/TERM/I/isochronous.html.
} 
worth mentioning that the paper is not meant to be a tool of comparison between the two communication systems but, rather, it aims at investigating the effects of the networks on the behavior of the overall application. To this end we will specifically evaluate the impact of the parameters and performance indicators of the networks on the tracking error indexes of the application.

In detail, the paper is organized as follows. Section 2 briefly discusses the employment of RTE networks for motion control applications and introduces the set of performance indicators. Section 3 provides the description of both EtherCAT and EPL as well as a theoretical analysis of their behavior. Section 4 introduces the coordinated motion control application. Section 5 analyzes the possible network configurations along with the scheduling policies. Section 6 presents the simulation results. In particular, after analyzing an ideal operational scenario, we simulate the behavior of the application under some typical error conditions such as, for example, jitter, electromagnetic interferences, physical layer errors. Section 7 concludes the paper and points out some future developments and research issues.

\section{Real-Time Ethernet Networks for Motion Control Applications}

According to [2], the three nested control loops (position, velocity and torque) of an electrical drive are typically embedded in the drive itself. Hence, when the connection between drive and controller is implemented by means of a communication network, the controller has to transmit the necessary set-points via the network. On the other hand, the standard explicitly foresees the possibility that some control loops are implemented on the controller (although such an alternative is just mentioned, in the standard, for future developments) resulting in a networked control system (NCS) [22]. Several studies have been actually carried out in the past years concerning NCSs, most of them were aimed at evaluating the effects of the networks on the overall control system performance. In some cases the networks employed were completely dedicated (sometimes even explicitly designed) for the NCS application as described, for example, in [23], where the authors developed a real-time closed loop control system with a sampling period of $600 \mathrm{~ns}$. Conversely, other analysis, even if not specifically related to motion control systems, have taken into consideration commercially available networks [24], [25], [26], [27].

Clearly, RTE networks may be profitably employed in NCSs as well, pro- 
vided that a tight synchronization with the application(s) running on the controller is maintained. This requires that the tasks actually implementing the control loops have to be scheduled in real-time by the operating system of the controller (interestingly, paper [25] actually focuses on such an issue). To this regard, from a practical point of view, it is worth mentioning that both the organizations supporting the two networks considered in this paper propose hardware/software products (e.g. PCs, PLCs, real-time operating systems, etc.) that allow to achieve the necessary synchronization.

In this paper, however, as previously mentioned, we refer to the traditional employment of the networks, meaning that they are used to deliver the setpoints but they do not implement data transfer within the control loops. Conversely, the contribution we propose starts from the analysis of the configurations and of the performance indicators. Then, we take into consideration some possible problems deriving from the adoption of RTE networks and evaluate their impact on the coordinated motion control application. Furthermore, we address some techniques that might mitigate the negative effects of the aforementioned communication drawbacks.

\subsection{Performance Indicators}

The IEC61784-2 standard defines a set of Performance Indicators (PIs) in order to specify the capabilities of the RTE networks [12]. In this section we recall some of the most relevant PIs, specified by the aforementioned standard and, moreover, we introduce two new PIs, namely Minimum Cycle Time and Jitter, which reveal particularly effective for both the specific networks considered when employed for coordinated motion control applications.

\subsubsection{PIs defined by IEC61784-2}

The PIs we consider are: Throughput RTE and Non-RTE Bandwidth. The Throughput RTE accounts for the ability of the network to handle realtime data. Indeed, it is defined as "the number of octets per second transmitted on a specific link exclusively relevant to APDUs traffic".

The Non-RTE Bandwidth represents the "percentage of the network bandwidth that can be used, on a specific link, for non-real time traffic".

\subsubsection{PIs specifically defined}

When a communication network is used in coordinated motion control applications, the set-point values are periodically transferred from the con- 
troller to the electrical drives. The actual transmission takes place in different ways, depending on the specific protocols adopted by the networks. The functioning of some RTE networks is based on a cycle during which data are transferred between a controller and some passive nodes such as, for example, sensors/actuators (both EPL and EtherCAT actually work according to this principle of operation, as it will be described in Section 3). In these networks, the set-points will be transmitted every cycle (or at multiples of a cycle) to the electrical drives. The duration of a cycle is a parameter typically known as "cycle time", which is set at the beginning of the network operation. On the other hand, the minimum time requested to execute a cycle represents a crucial indicator of the overall behavior of the system. For such a reason, we consider it as a performance indicator in this type of applications and refer to it as Minimum Cycle Time (MCT).

Furthermore, the duration of a cycle can be negatively affected by a certain degree of uncertainty due to several, possibly random, factors. Indeed, unpredictable transmission delays and/or errors (as described, for example, in [28] for a specific fieldbus) as well as latencies in the network stations and/or components, may result in a variable duration of the cycle time. Such a phenomenon is known as Jitter and we evaluate it by means of a further performance indicator defined as:

$$
J=\frac{\left|T_{C}-T_{C}^{m}\right|}{T_{C}}
$$

where $T_{C}$ is the desired cycle time (i.e. the value set at the beginning of network operation), whereas $T_{C}^{m}$ is the actually measured value. $J$ varies over the time and may be evaluated at every cycle by any of the network nodes.

\section{Ethernet POWERLINK and EtherCAT}

According to the IEC standardization, RTE networks are grouped in Communication Profile Families (CPFs), each of them including one or more Communication Profiles (CPs). In particular, Ethernet POWERLINK is specified by the CPF13 of IEC 61784, which contains only one CP (CP13/1). Similarly, EtherCAT is defined by CPF12 which contains two CPs.

\subsection{Background: Ethernet POWERLINK}

EPL is based on a data link layer protocol placed on top of legacy Ethernet medium access control sub-layer. The protocol relies on a master-slave 


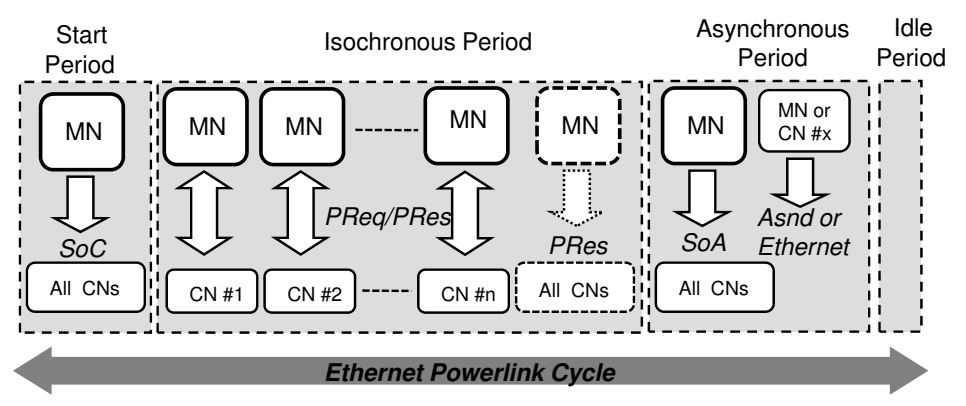

Figure 1: Ethernet POWERLINK Cycle

relationship and defines two types of stations: Managing Node (MN) and Controlled Nodes (CNs). The MN, which represents the controller, is unique within the network, whereas several CNs (up to 240) may be employed in various configurations. The interconnection between network devices may be realized either via hubs or switches ${ }^{2}$.

The MN polls periodically the CNs, realizing in this way the EPL cycle, described in Fig. 1, which is continuously repeated on the network. The cycle is started by the MN which broadcasts the Start of Cycle (SoC) frame. Then, the Isochronous Period is entered where the MN polls the CNs. In this phase, the MN issues a PReq (Poll Request) frame carrying the output data to each CN which, consequently, responds with a PRes (Poll Response) frame containing the input data. PRes frames are broadcasted on the network so that each station may read the input data transmitted by any CN. Once the CNs have been polled, the MN may optionally broadcast a PRes frame before the end of the Isochronous Period. Subsequently, the MN sends the SoA (Start of Acyclic) frame to notify the beginning of the Acyclic Period to the CNs. This frame allows to grant either a single $\mathrm{CN}$ or the MN itself with the access to the network for the transmission of only one acyclic message. According to the standard, two types of asynchronous frames may be transmitted, namely POWERLINK ASnd (Asynchronous Send) and Legacy Ethernet messages. Finally, the Idle Period is entered, where the MN waits for the remaining time before starting a new cycle.

According to [19], the duration of the EPL cycle can be trivially evaluated

\footnotetext{
${ }^{2}$ Actually the standard recommends the use of hubs since they ensure low and deterministic latencies.
} 
as the sum of the periods shown in Fig. 1:

$$
T_{C}^{e p l}=T_{s t}+T_{i s}+T_{a s}+T_{i d}
$$

In equation (2), $T_{s t}$ is the duration of the start period, expressed as the time necessary to send the SoC frame plus a safety margin necessary to ensure that all nodes are ready for the beginning of a new cycle. $T_{i s}$ and $T_{a s}$ are, respectively, the duration of the Isochronous and Asynchronous periods, $T_{i d}$ is the idle time.

Both $T_{i s}$ and $T_{a s}$ depend on the amount of data exchanged as well as on the network configuration. Specifically, the duration of the Isochronous Period can be expressed as:

$$
T_{i s}=T_{r q}+T_{r s}+D_{m n}+D_{c n}+D_{n k}
$$

where $T_{r q}$ is the time necessary to send all the PReq frames by the MN; similarly, $T_{r s}$ is the time employed by the CNs to send the PRes frames. Both these times depend on the overall number of CNs actually deployed as well as on the number of bytes they exchange with the MN during each EPL cycle. $D_{m n}$ and $D_{c n}$ are, respectively, the delays introduced by the MN and the CNs. For example, when the $\mathrm{MN}$ receives a PRes frame from a $\mathrm{CN}$, it has to wait for a "quiet" time before moving on the next CN (this time is referred as $t_{P R s-P R q}$ in [20] with a typical value of $1 \mu \mathrm{s}$ ). Finally, the term $D_{n k}$ in equation (3) accounts for the delay introduced by all the network components including, in particular, latencies and propagation times.

The duration of the Asynchronous Period is given by:

$$
T_{a s}=T_{s a}+T_{f r}
$$

where $T_{s a}$ is the time necessary to transmit the SoA frame and $T_{f r}$ is the time necessary to complete the acyclic frame transmission. This last term includes delays similar to those of equation (3). However, since the acyclic traffic may involve different stations at each cycle, and hence variable delays, we do not provide further details about $T_{f r}$, leaving them to a specific evaluation, if necessary.

A more comprehensive analysis of EPL is provided in [29].

From the above equations, it is interesting to observe that the minimum cycle time of EPL may be calculated from the knowledge of:

(i) the network configuration, which allows to evaluate the terms $D_{m n}$, $D_{c n}$ and $D_{n k}$ in equation (3); 
(ii) the number of bytes exchanged between $\mathrm{MN}$ and $\mathrm{CNs}$, which allows to calculate both $T_{r q}$ and $T_{r s}$ in equation (3);

(iii) an estimation of the asynchronous traffic which allows to calculate the term $T_{f r}$ in equation (4) and, consequently, the duration of the asynchronous period.

Finally, it is worth observing that not all the CNs are necessarily polled during an EPL cycle. Indeed, the EPL protocol specifies two communication classes, namely continuous and multiplexed that allow to differentiate the CNs to query cycle by cycle.

\subsection{Background: EtherCAT}

EtherCAT is a master/slave network which uses, at the data link layer, a "summation frame" technique similar to that employed by Interbus [30]. In practice, during the functioning of EtherCAT, a single frame periodically issued by the master station circulates among all the slaves. At the arrival of the frame, each slave extracts the output data addressed to it and inserts the input data for the master. These operations are carried out "on the fly", i.e. while the frame is crossing the slave and, hence, the frame itself is not received and subsequently retransmitted but, rather, it just passes through the slave. When the frame reaches the last slave, it is redirected towards the master as shown in Fig. 2, which reports an example of network configuration.

As can be seen, from a logical point of view, EtherCAT networks are based on a ring topology practically implemented by means of point-to-point connections between consecutive nodes. This requires that all the network stations (either master or slaves) are full-duplex devices capable of receiving/transmitting data concurrently. Moreover, the standard specifies the possibility of combining basic configurations with more complex ones such as stars: this is achieved by means of specific slave interfaces which allow to implement branches.

EtherCAT uses standard Ethernet frames which, similarly to EPL, encapsulate the telegrams specifically defined by the protocol, as shown in Fig. 3. This allows, from an implementation point of view, that the master is a common Ethernet node running the EtherCAT protocol. Conversely, the "on the fly" elaboration which has to be provided by the slaves, requires that these devices are equipped with special hardware components which make them quite different from legacy Ethernet nodes. 


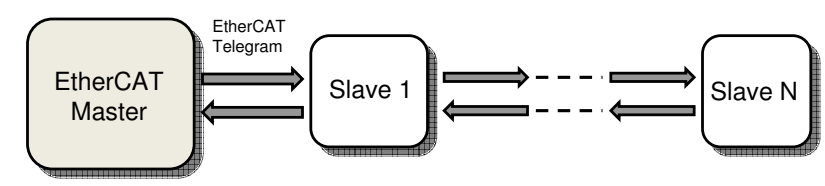

Figure 2: EtherCAT Configuration

Fig. 3 also shows the structure of the EtherCAT telegrams contained in the Ethernet data field. As can be seen, a telegram begins with a 10-byte header that, in particular, specifies the type of operation for which it is intended (for example read, write and read/write). The header is followed by the data field which represents the area in which slaves write and/or read plant data during the network operation. Finally, the working counter is a 2-byte field employed by the master to check for the correct execution of the operation specified in the header field. Typically, EtherCAT telegrams are employed for exchanging real-time data between master and slaves. However, EtherCAT networks are able to cope with non real-time traffic as well. This takes place by means of mailboxes, which are specific structures that may be fitted within an EtherCAT telegram. In this way, for example, a TCP/IP protocol data unit may be inserted in a mailbox.

EtherCAT makes use of an interesting technique, namely the logical addressing, in order to implement an effective data exchange between master and slaves. With such a mechanism, slaves are not directly addressed by the master but, rather, they read/write actual plant data from/to a logical memory. In practice, each slave has assigned a set of fieldbus memory management units (FMMUs) which are logical structures directly mapped on the slave physical input/output data. In an EtherCAT telegram, the master may specify a logical address referring to several FMMUs, possibly located on different slaves. During operation, each slave analyzes the logical address of the frame which is passing through: if the address matches with one or more of its FMMUs, then that slave extracts the output data and writes the input ones. In this way, a unique header (telegram) may be used to address several slaves with the consequent improvement of the protocol efficiency.

An EtherCAT master, in principle, may issue telegrams with different periods, possibly addressing different sets of slaves. Clearly, the time necessary to transmit a telegram represents the minimum period of transmission of that telegram and hence, according to the definition given in Section 2, the minimum cycle time. From the structure of an EtherCAT frame shown in 


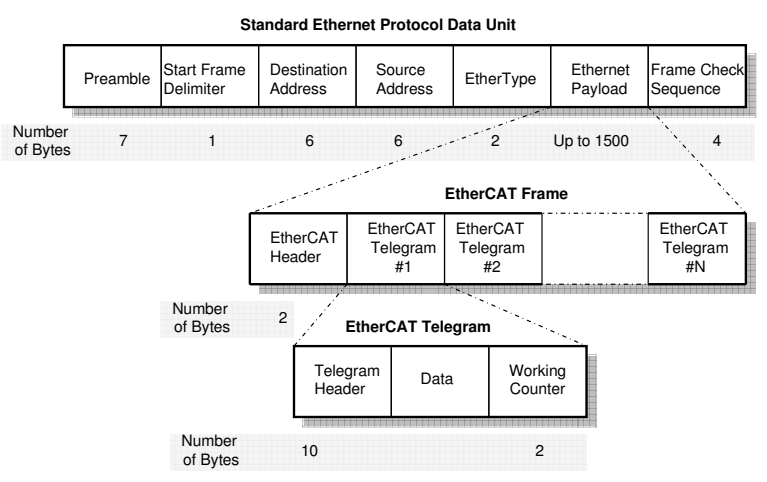

Figure 3: Structure of the EtherCAT Frame

Fig. 3 as well as from the theoretical analyses provided in [31] and [32], the cycle time may be expressed as:

$$
T_{C}^{e t c}=T_{e t}+T_{e c}+T_{d e}+T_{i f}
$$

where, $T_{e t}$ is the time necessary to transmit all fields of the frame but the payload (26 bytes in total, which require $2.08 \mu \mathrm{s}$ at $100 \mathrm{Mbits} / \mathrm{s}$ ); $T_{e c}$ is the time actually requested for transmitting the EtherCAT frame, which is encapsulated in the payload of the Ethernet frame; $T_{d e}$ is the delay introduced by the slaves due to the elaboration of the frame: assuming the same delay for each slave ${ }^{3}, T_{s v}$, then trivially we have: $T_{d e}=N \times T_{s v}$, where $N$ is the number of slaves; finally, $T_{i f}$ is the time which has to be waited before issuing a new frame (referred as "interframe gap" in the original Ethernet specification: $0.96 \mu \mathrm{s}$ at $100 \mathrm{Mbits} / \mathrm{s})$.

In equation (5) the term $T_{e c}$ is specifically related to the EtherCAT protocol and, as such, it can be further analyzed. In particular, it is given by:

$$
T_{e c}=T_{e h}+L \times\left(T_{t h}+T_{w c}\right)+\sum_{i=1}^{L} T_{c t}^{(i)}
$$

where $T_{e h}$ is the time necessary to transmit the EtherCAT header; $L$ is the number of EtherCAT telegrams within the frame; both $T_{t h}$ and $T_{w c}$ account for the transmission of, respectively, the telegram header and the working

\footnotetext{
${ }^{3}$ this is reasonable, since different slave devices likely use the same hardware for frame elaboration.
} 
timer; finally, $T_{c t}^{(i)}$ is the time requested to transmit the data field of the $\mathrm{i}-\mathrm{th}$ EtherCAT telegram in the frame.

\section{Coordinated Motion Control Application}

In order to provide some insights on the use of RTE networks, we considered, as a test bench, the coordinated two-axis motion control. The application, quite common for this kind of tests, was the tracking of a circular trajectory by the coordinated motion of two independent axes. The references, two sinusoidal functions in quadrature with period $50 \mathrm{~ms}$, were transmitted by the controller through the RTE networks. The received data were then collected in a file which was sequentially accessed by the two drives, at a fixed rate of $500 \mu s$, implementing a direct velocity control technique scheme, as defined in [2] and pointed out in [11]. Each axis was driven by a surfacemounted permanent magnet synchronous motor (PMSM-SPM), whose most significant data are reported in Table 1.

Table 1: PMSM parameters

\begin{tabular}{|c|c|}
\hline Parameter & PMSM \\
\hline \hline Nominal speed $\omega_{N}$ & $4000 \mathrm{rpm}$ \\
\hline Nominal torque $\tau_{N}$ & $0.87 \mathrm{Nm}$ \\
\hline Pole pairs $p$ & 2 \\
\hline Stator resistance $R_{s}$ & $1.47 \Omega$ \\
\hline Synchronous inductance $L_{s}$ & $5.17 \mathrm{mH}$ \\
\hline PM flux linkage $\Lambda_{P} M$ & $0.066 \mathrm{Vs}$ \\
\hline
\end{tabular}

The block schematic of one axis is reported in Fig. 4. The coordinated motion control application has been simulated by means of two different numerical tools, namely Opnet [33] and Simulink [34]. In particular, Opnet simulation models have been derived for both the RTE networks, whereas the behavior of the electrical drives has been obtained via Simulink.

In principle, the trajectory tracking can be considered the classical task of a positioning routine, which incorporates the nested velocity and torque closed 


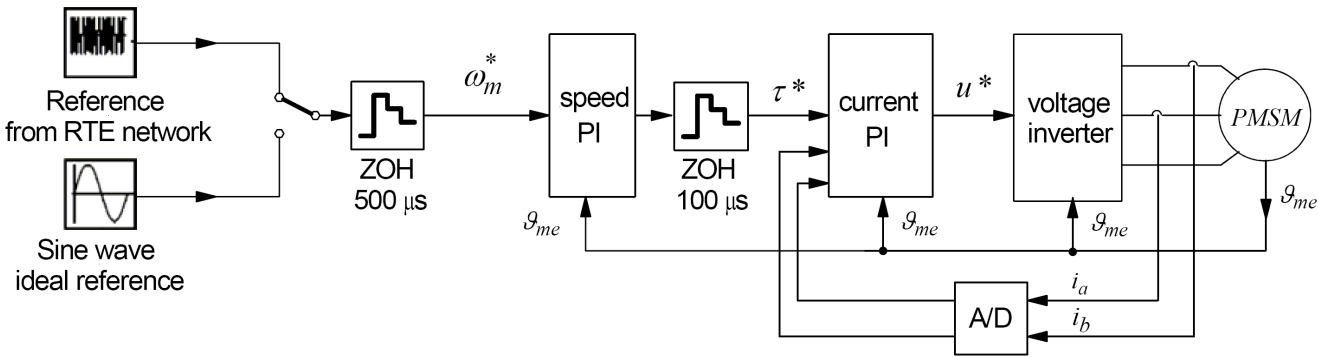

Figure 4: Coordinated motion - Structure of the x-axis PMSM drive

loop controls. On the other hand, in our case the synthesis of the position control algorithm, with its manifold variants and practice-related implementation hints, could negatively affect the intelligibility of the analysis, which aims at being as much as possible independent of the electrical drives. To this extent, and without lack of generality, in the present work the reference data have been considered as two velocity references, which directly feed the velocity control loops. For a closer adherence to industrial standards, the simulation considers full digital algorithms. The Bode diagrams of both velocity and current loops are shown in Fig. 5a and Fig. 5b. The two control cycles for velocity and current loops were of $500 \mu s$ and $100 \mu s$, respectively. As a consequence, in order to correctly deliver the velocity set-point samples, assuming that the drives receive the set-point from the controller at every cycle, then the minimum cycle time of both the RTE networks has to be equal to or lower than $500 \mu s$.

The velocity bandwidth was about $140 \mathrm{~Hz}$, while that of the inner current loop was about $600 \mathrm{~Hz}$. The simulation returns the actual position of the payload, which was an inertial load of $95 e^{-} 6 \mathrm{kgm}^{2}$. In order to calibrate the simulation model, the tracking error indexes (which will be defined in Section 6) were first computed when the system was fed from an ideal couple of built-in references. In that case, inaccuracies were inherently due to the drive control implementation only. Then, different data files, obtained from the RTE networks data transmission, were tested in sequence.

\section{Network Configurations}

In order to test the performance of the coordinated motion control application we refer to network configurations commonly employed in factory 

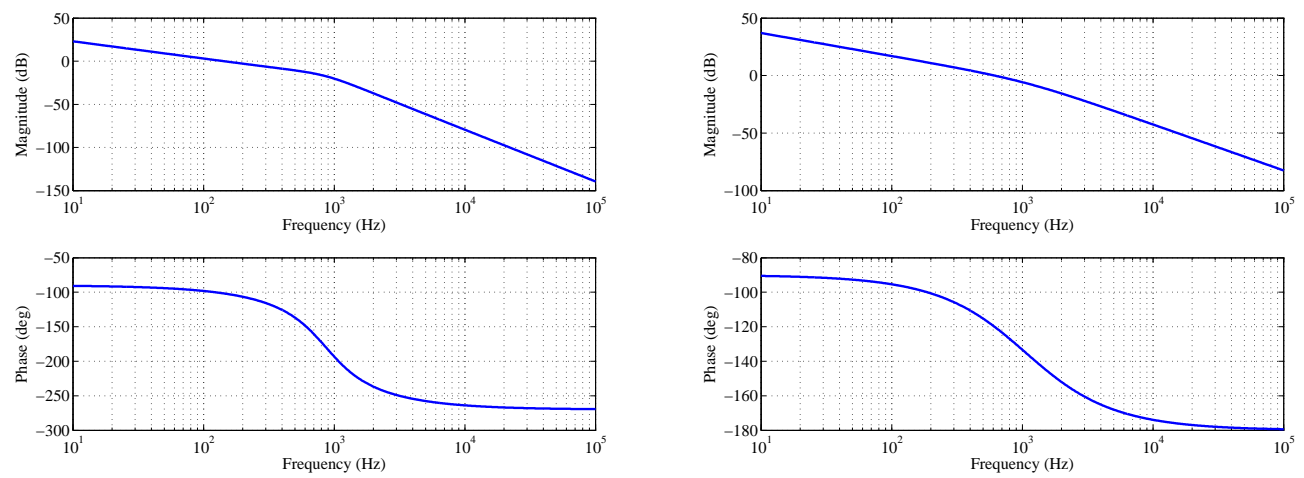

(a)

(b)

Figure 5: Bode plots of the velocity loop (a) and the current loop (b).

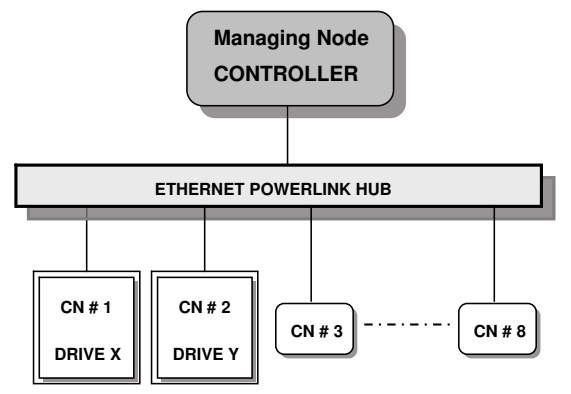

Figure 6: Ethernet POWERLINK Configuration

automation systems. In particular, as described in [35], we consider a onelevel configuration comprising, in our case, one controller device, the two electrical drives and six additional passive nodes ${ }^{4}$. Clearly we are conscious that different alternatives are possible, nonetheless, the chosen configuration is widespread and, as such, it represents a suitable testbed for the application. The configuration of EPL is shown in Fig. 6. As can be seen, it employs a single hub and the two electrical drives are the first two CNs of the network. Similarly, we use an equivalent linear configuration for EtherCAT, where the drives are the first two slaves. The velocity set-points are expressed as 32-bit size floating point values and we assume that each drive

\footnotetext{
${ }^{4}$ a passive node may be either an EPL Controlled Node or an ETherCAT slave
} 
sends back to the controller four bytes of input data; moreover, considering that real-time traffic in industrial applications is characterized by the transfer of limited amounts of data with tight deadlines [14], we suppose, without loss of generality, that each slave exchanges four input bytes and four output bytes of real-time data with the controller (for the sake of clarity, to this regard, it is worth pointing out that other types of sensors, like for example cameras, have recently started to be included in the control loops [36] and, in these cases, the amount of data they exchange may be considerably greater). Nonetheless, these types of applications are currently quite unusual. Finally, we suppose the presence of non real-time traffic as well; in particular, a 200byte TCP/IP Protocol Data Unit is sent, once per network cycle, by a specific slave which acts as a gateway towards an office network. The parameters of the communication application are summarized in Table 2 .

Table 2: Communication Parameters

\begin{tabular}{|c|c|}
\hline \hline Total Number of Passive Nodes & 8 \\
\hline Input Bytes Exchanged per Passive Node & 4 \\
\hline Output Bytes Exchanged per Passive Node & 4 \\
\hline NRT Bytes Exchanged per Network Cycle & 200 \\
\hline \hline
\end{tabular}

\subsection{EPL Scheduling}

In order to select an appropriate scheduling of the CNs, Table 3 reports the typical values of the most relevant parameters as specified by the standard document.

Given the amount of bytes exchanged between MN and CNs reported in

Table 3: EPL Parameters

\begin{tabular}{|c|c|c|}
\hline \hline Parameter & Meaning & Value \\
\hline \hline$t_{P R s-P R q}$ & Waiting Time of MN per each CN & $1 \mu s$ \\
\hline$t_{P R q-P R s}$ & Response Time of each CN & $8 \mu \mathrm{s}$ \\
\hline$T_{s t}$ & Duration of the Start Period & $45 \mu \mathrm{s}$ \\
\hline \hline
\end{tabular}


Table 2, the PReq/PRes frames result minimum size Ethernet frames (512 bits), with a corresponding transmission time (at $100 \mathrm{MBits} / \mathrm{s}$ ) of $5.12 \mu \mathrm{s}$. As a first natural choice, we may assume that all CNs are polled in each cycle (the standard refers to such a feature as "continuous communication class"). Consequently, in eq. (3), both $T_{r q}$ and $T_{r s}$ result $40.96 \mu s$; moreover, from the EPL parameters provided in Table 3 we have $D_{m n}=8 \mu s$ and $D_{c n}=64 \mu \mathrm{s}$. Hence, neglecting the term $D_{n k}$ (the propagation times and the delay introduced by the hub are, at least, one order of magnitude below the other terms in eq. (3)), we obtain for the isochronous time: $T_{\text {is }}=$ $153.92 \mu \mathrm{s}$. The asynchronous period needs to be at least $22.08 \mu \mathrm{s}$ (necessary to transmit both the SoA frame and the TCP/IP message). It turns out that, considering the duration of the start time, the minimum cycle time for $\mathrm{EPL}$ is $M C T^{e p l}=221 \mu \mathrm{s}$.

Clearly, different scheduling schemes are possible, thus in this context we analyze two additional specific solutions that could reveal interesting for the coordinated motion control application.

\subsubsection{Multiplexed Time Slots}

As an alternative to the continuous polling, depending on the communication requirements, only a subset of the CNs may be queried within an EPL cycle ("multiplexed communication class"). For example, in each cycle we could poll both the drives and only one of the other CNs (which is changed cycle by cycle). This would require six consecutive cycles to execute a complete query of all the $\mathrm{CNs}$, but in this case, the minimum cycle time reduces to $M C T^{e p l}=124.8 \mu \mathrm{s}$. Alternatively, only two nodes could be polled per cycle, so that four cycles are necessary for the complete query, leading to $M C T^{e p l}=105.56 \mu \mathrm{s}$. It is worth observing that, with such a scheduling, both the drives are polled once every four cycles but, given the requirements of the coordinated motion control application (sampling time $=500 \mu \mathrm{s}$ ), the timed delivery of the set-points is still ensured.

\subsubsection{Multicast Addresses}

As a further scheduling scheme that allows to obtain shorter cycle times, multicast addresses could be employed. Such a technique is actually already used by EPL, but only for a specific set of messages (for example the Start of Cycle, the Start of Asynchronous and the PRes frames are broadcasted to all the nodes), consequently, its actual introduction would require a slight modification to the EPL protocol which, however, maintains total compatibility 
with the original version. The proposed modification consists basically in the assignment of multicast addresses to groups of CNs; in such a way, the PReq frame issued by the MN to a multicast address is received by all the CNs belonging to that address. Clearly, the CNs are not allowed to reply with the PRes frames, since these latter would likely collide on the network. Thus, the technique can be used by $\mathrm{CNs}$ that just need to receive data from the MN without replying to it. Hence, in particular, it is suitable for the transmission of the set-point values to the electrical drives. In the coordinated motion control application, for example, both the drives could be grouped in a multicast address; then the MN, within the EPL cycle, sends a single (minimum size) PReq frame containing the two set-points to the multicast address $^{5}$. Thus, if for example the two drives and only one CN are polled in each EPL cycle, the minimum cycle time results: $M C T^{e p l}=92.44 \mu \mathrm{s}$.

\subsection{EtherCAT Scheduling}

The EtherCAT scheduling has been designed assuming that a single frame is employed comprising two EtherCAT telegrams: the first one is used for the real-time data, whereas the second one contains the mailbox which, in turn, accommodates the TCP/IP protocol data unit. From Fig. 3 and considering also the communication parameters of Table 2, the EtherCAT telegram \#1 results 76 bytes long, whereas the EtherCAT telegram \#2 results 212 bytes long.

Clearly, the minimum EtherCAT cycle time is represented by the time necessary to transmit the frame containing the two telegrams. Its evaluation, however, requires knowledge of the delay introduced by each slave, $T_{s v}$. This delay, actually, is not specified by the standard whereas, in [31], a value of $1.35 \mu \mathrm{s}$ is indicated when working at $100 \mathrm{Mbits} / \mathrm{s}$, but without an adequate explanation of the choice. Thus, we resorted to experimentally evaluate such a value and, consequently, we built up a suitable testbed comprising a set of commercially available devices. From the practical tests executed, we measured an average delay of $1 \mu s$ and, hence, such a value has been used to calculate $T_{d e}$ in eq. (5). Thus, in eq. (5) we have $T_{d e}=8 \mu \mathrm{s}$ and $T_{e c}=23.2 \mu \mathrm{s}$. As a conclusion, the minimum cycle time of the EtherCAT network results $M C T^{e t c}=34,24 \mu s$.

\footnotetext{
${ }^{5}$ In this case the communication parameters are changed from those reported in Table 2 since there are not input bytes sent from the drives to the MN.
} 


\subsubsection{Performance Indicators Calculation}

In order to evaluate the most relevant performance indicators, we set the cycle time of both the networks to $500 \mu s$, since this value is the sampling time of the coordinated motion control application. Clearly, lower cycle times could be set and, actually, such a possibility will be further investigated in the next section, since it allows to obtain better performance of the application. As far as EPL is concerned, according to the above analysis, we considered three different types of scheduling, namely continuous (all CNs polled during a cycle), multiplexed (both the drives plus one different CN polled per cycle) and multicast (both the drives grouped in a multicast address plus one different $\mathrm{CN}$ polled per cycle). With regard to EtherCAT, the scheduling scheme described in the previous paragraph represents the most effective choice and, as such, it has been addressed.

The performance indicators defined in Subsection 2.1 calculated for both EPL and EtherCAT are reported in Table 4 (the terms "EPL-cnt", "EPLmtx" and "EPL-mtc" refer, respectively, to the continuous, multiplexed and multicasted scheduling schemes of EPL).

Table 4: Performance Indicators

\begin{tabular}{|c|c|c|c|c|}
\hline \hline PI & EtherCAT & EPL-cnt & EPL-mtx & EPL-mtc \\
\hline \hline MCT $[\mu s]$ & 34.24 & 221 & 124.8 & 92.44 \\
\hline Thrpt. RTE $[\mathrm{kB} / \mathrm{s}]$ & 233.6 & 36.2 & 64.1 & 151.4 \\
\hline Non RTE Bdw. [\%] & 96.6 & 60.2 & 79.45 & 85.9 \\
\hline \hline
\end{tabular}

The minimum cycle time values shown in Table 4 have been evaluated in the previous paragraphs.

The calculation of the Throughput RTE has been made considering the actual RTE data (in bytes) exchanged during the minimum cycle time; moreover, since the definition of such a performance index refers explicitly to the RTE traffic on one specific link, we considered, for both the networks, the connection between the controller and the first passive device (which, however, has the same RTE throughput of all the other links, since in our example each slave exchanges eight bytes with the controller). The lower Throughput RTE values of EPL (compared with EtherCAT) may be partially explained by the limited amount of data transmitted with the PReq/PRes frames that, in any case, have to be transferred by minimum size Ethernet frames. 
The Non RTE Bandwidth, according to its definition, has been calculated as the percentage of bandwidth within a cycle that may be used for non real time traffic. Thus, for example, with regard to the continuous polling scheme of EPL, given the cycle time $(500 \mu s)$, the time available for acyclic traffic from eq.(2) results $T_{C}-\left(T_{s t}+T_{i s}\right)=301.08 \mu \mathrm{s}$. Similarly, as far as EtherCAT is concerned, the time available for non real-time traffic is given by the the difference between $T_{C}$ and the time necessary to transmit the EtherCAT telegram \#1 (17.04 $\mu s)$ resulting $482.96 \mu s$.

\section{Performance Analysis}

In order to evaluate the performance of the proposed application we refer, as tracking error indexes, to the set of purposely defined performance indexes for quality response of dynamic systems cited in [37]. In particular, from the definition of error $e(t)$, as the difference between the reference value, $r(t)$, and the actual system response, $y(t)$, we consider both the Integral of Absolute Error, IAE, and the Integral of the Square Error, ISE, defined as follows:

$$
I A E=\int_{0}^{\infty}|e(t)| d t ; \quad I S E=\int_{0}^{\infty} e^{2}(t) d t .
$$

It is worth observing that IAE accounts for the cumulative error, whereas ISE is more concerned with the energy of the error signal since it gives more weight to larger errors.

\subsection{Tests under Ideal Conditions}

From Table 4 it results that the minimum cycle times of both the networks are well below the sampling period of the coordinated motion control application. Consequently the networks are, at least in principle, adequate to satisfy the requirements of the considered automation system.

In a first step, we checked the behavior of the application under ideal operational conditions, meaning that all the set-point values were periodically delivered by the controllers and they were correctly received by the electrical drives.

We simulated the operation of 10 periods of the application, resulting in an overall running time of $500 \mathrm{~ms}$ (i.e. 1000 network cycles). The outcomes of the simulation showed that the circular trajectory was tracked correctly, as confirmed by the tracking error indexes that resulted both equal to zero. 
This result corroborates the effectiveness of the proposed architecture and, in particular, it confirms that the delivering of the set-points by means of the RTE networks does not imply any negative influence on the behavior of the coordinated motion control application.

\subsection{Effects of Increasing the Network Load}

From the aforementioned simulations, and considering the scheduling policies discussed in Section 5, it may be definitely concluded that the coordinated motion control application works appropriately if the networks are able to cope with the sampling period imposed by the application. Hence, in practice, it is necessary that the minimum cycle time of the networks is lower than the sampling period of the application. However, if the network load increases, as an immediate effect, the minimum cycle time increases too, limiting in such a way the performance of the network. Thus, it reveals interesting, for a given network configuration, to evaluate the behavior of the minimum cycle time versus the actual network load. For the applications of interest, the load of the networks may grow due to two specific reasons, namely, the addition of new nodes (either electrical drives or passive devices) and/or the increase of the input/output bytes exchanged between the controller and the other nodes. In order to provide an example in this direction, we plotted the behavior of the minimum cycle time for the networks described in the previous Section as a function of the offered load. The calculation of the MCT has been carried out following the analysis provided in Section 3. In particular, two behaviors are provided. In the first one, shown in Fig. 7a, the load is increased adding new passive nodes. We referred to the network configuration provided in Fig. 6 which comprises an increased (with respect to the figure) fixed number of drives ( 8 in total, each exchanging 4 bytes with the controller) and a variable number of generic passive nodes whose communication features are reported in Table 2. For both the multiplexed and multicast scheduling of EPL shown in Fig. 7a, we assumed that during one EPL cycle all the drives and one CN were polled. Such a choice ensures a constant value of the minimum cyle time of EPL at the expenses, however, of a longer polling period of the CNs. The second behavior, shown in Fig. 7b, refers to a network with a fixed configuration comprising, namely, 8 drives and 8 generic passive devices. In this second example, the number of bytes exchanged between the controller and the drives is maintained constant ( 4 bytes, also in this case, representing the velocity set-point) whereas 
the generic passive nodes are supposed to exchange a progressively increased amount of I/O bytes. The same assumptions of the first example were used for both the multiplexed and multicast scheduling of EPL (all the drives plus one $\mathrm{CN}$ polled per cycle). In this case, as can be seen, the minimum cycle time of EPL remains constant for values of exchanged I/O bytes smaller than 41, since minimum size Ethernet frames are used (actually, up to 44 data bytes could be transmitted with a minimum size Ethernet frame, but the EPL protocol adds 3 bytes to the Ethernet payload).

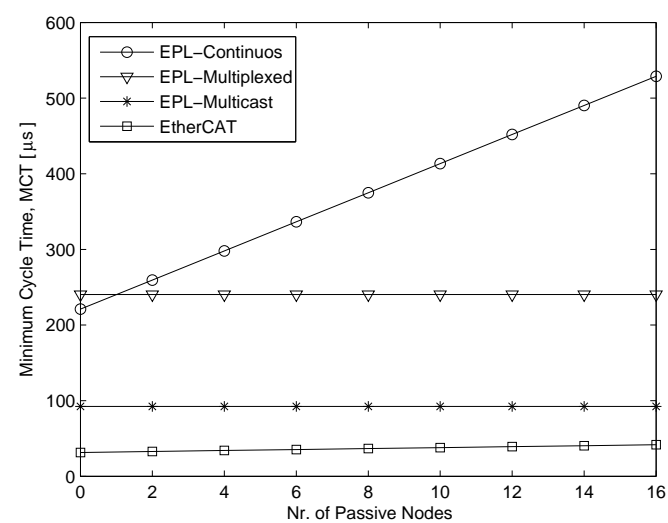

(a)

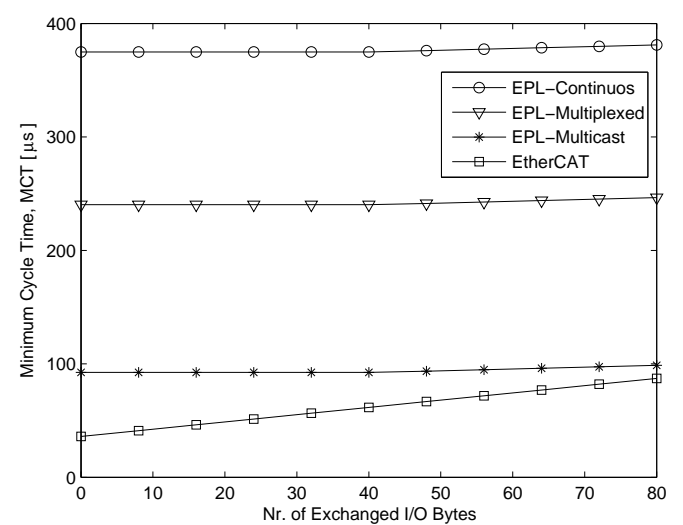

(b)

Figure 7: MCT vs Nr. of Passive Nodes (a) and vs Nr. of Exchanged I/O Bytes (b).

\subsection{Tests in Presence of Jitter}

Since the presence of jitter is one of the problems that may affect RTE networks, we studied the effects of such an inconvenient in the coordinated motion control application. The behavior of the networks considered may by influenced by the presence of jitter in two different situations: at the beginning of a cycle and during its execution.

Actually, there may be jitter at the beginning of a network cycle due to several causes. For example, if the current EPL cycle for whatever reason takes more than its expected duration, then the next one will be necessary delayed. Analogously, a task running on the EtherCAT master which exceeds its execution deadline, if not adequately preempted, may cause a delayed delivering of the EtherCAT frame, with the consequent jitter in updating the slaves. 
Furthermore, in EPL networks, jitter may appear during the execution of a cycle as well. Indeed, if for example a $\mathrm{CN}$ takes a longer interval of time to respond to the query of the MN (or, worse, it experiences a time out in issuing the PRes frame), then the next $\mathrm{CN}$ will be polled with an actual period greater than the previous one. This latter type of inconvenient can not occur in EtherCAT networks, since the dedicated hardware implementing the "on the fly" elaboration prevents the introduction of random delays by the slave devices.

As a consequence, whatever being the type of jitter, the set-point values may be delivered to the electrical drives at different instants than those expected. Since in the direct velocity control the drives periodically read the set points from the input buffers, it may happen that, erroneously, the same value is sampled twice or more times due to the late arrival (caused by jitter) of the current one sent by the controller. Clearly, the more the jitter, the higher the number of set point samples that can be missed by the drives. However, since in our application the drives get the set-point values periodically every $500 \mu \mathrm{s}$, the missing of more than one sample would be possible only in case the delay of the transmission is greater than such a value and, considering the cycle times of the two networks, this is very unlikely.

As a first step we simulated the behavior of the coordinated motion control application in presence of jitter. In particular, we assumed that $10 \%$ of the cycles were affected by jitter and that this causes the missing of just one sample. We considered two cases: the first one refers to jitter occurring at the beginning of the cycle, and hence common to both the networks (the simulation results, however, are obtained for the EtherCAT network), the second one concerns to jitter occurring within a cycle and hence relevant to EPL. We also assumed that, in this case, the jitter influences the electrical drive controlling the $\mathrm{y}$-axis.

The values of the tracking error indexes are reported in Table 5 for the two axes where, in the second column, the term "Both" means that the results have been simulated (and obtained) for both the networks, "ECAT" means that they are relevant to EtherCAT and "EPL" means that they are relevant to Ethernet POWERLINK. Analogously, in the third column, "B" stands for jitter at the beginning of a cycle, whereas "W" stands for jitter within a cycle.

As can be seen, the tracking error indexes have been calculated for two different values of the sampling time $(500 \mu s$ and $100 \mu s)$ used by the networks to periodically deliver the set-points. Such a technique is referred as 
Table 5: Tracking Error Indexes in Presence of Jitter

\begin{tabular}{ccccccc}
\hline \hline Samp. Int. & Netw. & J & IAE-X & IAE-y & ISE- & ISE-y \\
\hline \hline $500 \mu s$ & Both & B & 0.0032 & 0.0030 & 0.00005 & 0.00004 \\
$100 \mu s$ & ECAT & B & 0.0024 & 0.0023 & 0.00002 & 0.00002 \\
$500 \mu s$ & EPL & W & 0.0022 & 0.0030 & 0.00002 & 0.00002 \\
\hline \hline
\end{tabular}

oversampling in [24]. To this regard, results are shown in Table 5 for EtherCAT. However, similar performance may be achieved by EPL since, as shown in Table 4, the minimum cycle time values allow to set the duration of the EPL cycle down to $100 \mu s$ as well.

As a general consideration, the tracking error indexes have low values, as also proved by a graphical analysis we carried out, which did not show any visible flaw in the drawn circle. Moreover, the reduction of the sampling period reveals an effective technique to further reduce the error since, as expected, the faster the set-point samples are delivered, the lower the probability that the electrical drive, which reads the samples at a constant rate, bound to its bandwidth, may be affected by transmission errors.

In a further simulation analysis, we tested the behavior of the coordinated motion control application for increasing jitter values. Specifically, the mean values over the two axes ( $\mathrm{x}$ and $\mathrm{y}$ ) of both the error indexes, IAE and ISE, have been evaluated. Results are shown in Fig. 8 where the horizontal axis reports the percentage of network cycles that are influenced by jitter. Simulations have been carried out for an EtherCAT network (hence with jitter affecting the beginning of the cycles) with sampling rates of, respectively, $500 \mu s$ and $100 \mu s$. It can be observed, in general, as the coordinated motion control application is able to cope with the considered jitter values, since the tracking errors are kept low, ensuring the correct drawing of the circle. Furthermore, Fig. 8 shows once again the positive effect of the oversampling technique that makes this application practically insensitive to the jitter. As a final consideration, it is worth observing that the presence of jitter, due to its very short duration, is not detectable by the drives in any way and, hence, its effects are not recoverable.

\subsection{Test in Presence of Transmission Errors}

Industrial environments are typically characterized by the presence of several sources of electromagnetic interferences which may affect the functioning 


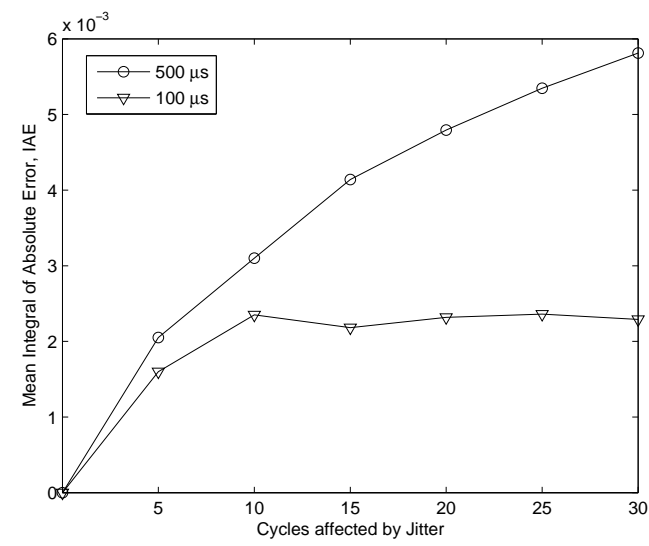

(a)

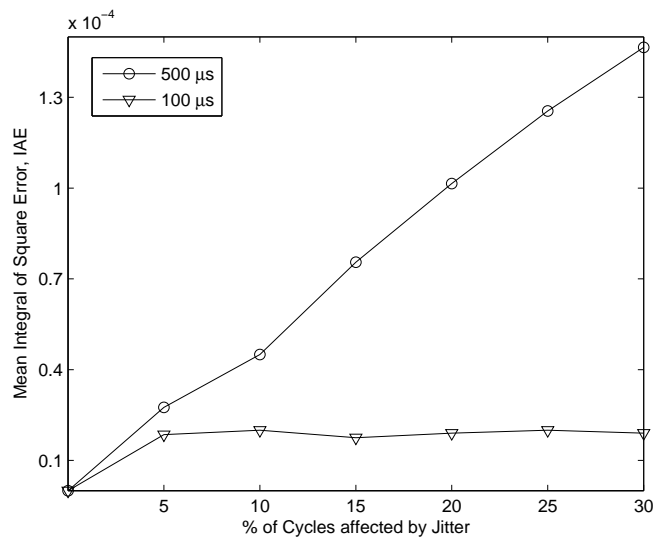

(b)

Figure 8: IAE vs Jitter (a) and ISE vs Jitter (b).

of electronic equipment and, specifically, that of communication networks causing in particular the corruption of frames. Both the RTE networks considered in this paper adopt a technique of frame error detection based mainly on the check of the FCS field of the Ethernet frames employed. The reactions of the networks at the occurrence of FCS errors are different; for example, an EPL CN enters a pre-operational state (where it temporarily stops data exchange) and waits to be re-identified by the MN. Conversely, an EtherCAT slave marks the frame as non valid and it does not increase its working counter, so that to be identified by the master. However, focusing on the coordinated motion control application, as a common consequence, if a drive receives a corrupted frame, it discards it, missing in such a way the updating of the set-point.

In order to provide some insights concerning the behavior of the application in the presence of interference, it is essential to know the characteristics of the noise which may affect the communication system. This is a critical (if not impossible) task, since it is related to the specific installations as well as to possibly time-changing environmental conditions. Anyway, as a general important result, some studies (for example those reported in [38], [39] and the references therein) pointed out that typically electrical noise occurs as bursts, so that the most suitable model for communication systems employed in industrial environments is a two-state channel often referred as GilbertElliot model [40]. According to such a model, a network may be in one of 
two states: bad and good. The bad state is characterized by a high error probability; conversely, in the good one the error probability is very low.

To select a suitable source of interference for the performance analysis, we actually considered, as a possible reference, the Electromagnetic Compatibility Standard [41] which specifies, as noise signal, a burst of $15 \mathrm{~ms}$ duration with a period of $300 \mathrm{~ms}$. However, since we are mainly interested on the effects of the noise within one period of the application, we supposed that the burst signal occurs, on average, every $50 \mathrm{~ms}$ (which is the period of the coordinated motion control application) and that the duration of the burst was as an uniform random variable ranging from $1 \mathrm{~ms}$ to $5 \mathrm{~ms}$. Clearly, depending on the characteristics of the environment, several other alternatives are possible, nonetheless we believe that the proposed scenario represents an effective benchmark.

In agreement with the previous simulations, we analyzed 10 periods of the application; moreover, we supposed that all the frames transmitted in the bad states were corrupted and that, in the good states, all the transmissions were completed successfully. In a first session of numerical simulations, we considered two specific scenarios, accounting for transmission errors occurring on both the electrical drives and on one single drive (controlling the $\mathrm{y}$-axis) respectively. Also in this case we used two different values for the sampling time of the RTE networks $(500 \mu s$ and $100 \mu s)$. The tracking error indexes are shown in Table 6 , where, as in the previous subsection, the results for the sampling time of $500 \mu s$ are common to both the networks whereas the results at $100 \mu s$ are relevant to EtherCAT. Moreover, in Table 6 , the column "Drive" indicates the number of electrical drives in which we actually simulated the occurrence of transmission errors.

Table 6: Tracking Error Indexes in Presence of Transmission Errors

\begin{tabular}{ccccccc}
\hline \hline Samp. Int. & Netw. & Drive & IAE-x & IAE-y & ISE-x & ISE-y \\
\hline \hline $500 \mu s$ & Both & 2 & 0.0073 & 0.0099 & 0.0012 & 0.0023 \\
$100 \mu s$ & ECAT & 2 & 0.0052 & 0.0062 & 0.0005 & 0.0010 \\
$500 \mu s$ & Both & 1 & 0.0022 & 0.0060 & 0.00002 & 0.0010 \\
$100 \mu s$ & ECAT & 1 & 0.0022 & 0.0058 & 0.00002 & 0.0009 \\
\hline \hline
\end{tabular}

As can be seen, the tracking error indexes are considerably greater than in the previous case indicating that the effects of transmission errors may 
be relevant for the whole application. This is also graphically evident, as shown in Fig. 9, which reports the drawn circle obtained from simulation with transmission errors affecting both the axes.

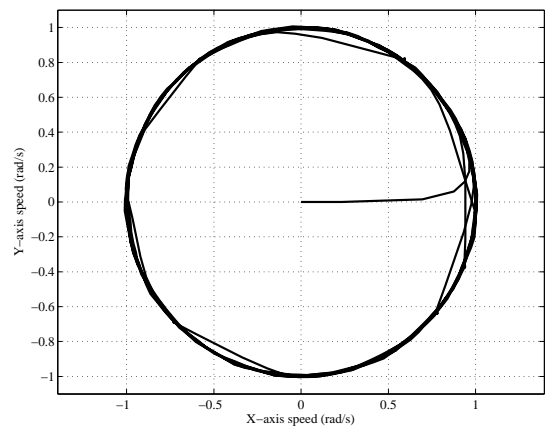

(a)

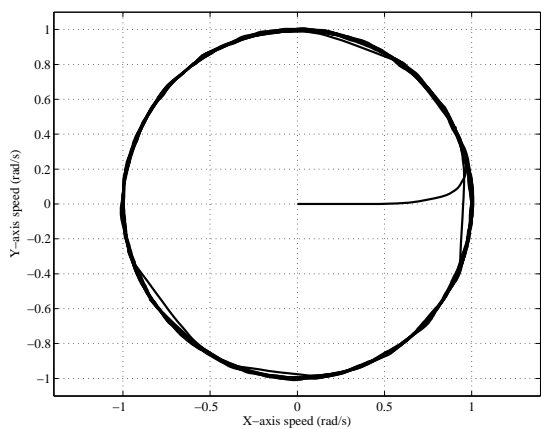

(b)

Figure 9: Drawn Circle with Sampling Time $500 \mu s$ (a) and $100 \mu s$ (b).

In a second simulation session we took into consideration longer burst durations (still modeled as uniform random variables), maintaining the same repetition period $(50 \mathrm{~ms})$. We referred specifically to an EtherCAT network with sampling rates of, respectively, $500 \mu \mathrm{s}$ and $100 \mu \mathrm{s}$, assuming that the errors occurred on both the drives. Results are provided in Fig. 10 where the horizontal axis reports the mean value of the error burst duration. Clearly, there is an evident performance worsening for longer bursts, since the tracking errors assume relevant values, with the consequent degradation of the drawn circle shape. On the other hand, both Fig. 9 and Fig. 10, along with Table 6 , show how such a degradation is mitigated by the oversampling technique.

\subsection{Physical Layer Errors}

These types of errors are mainly related to problems that may derive from the hardware components used by the RTE networks. Typical examples are cable breaks, defective connectors, faulty hub/switch devices, malfunctioning of Ethernet controllers, etc. In general these are permanent faults that seriously compromise the behavior of the application. Although the diagnostic techniques adopted by both the RTE networks allow to detect the occurrence of physical layer errors, they are not sufficient to ensure adequate recovery procedures for the coordinated motion control application. Focusing, for example, on EPL, a cable break between the MN and one of the two 


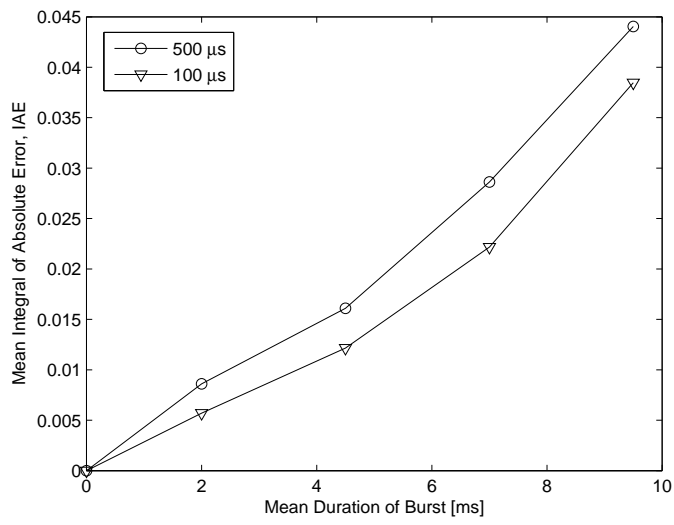

(a)

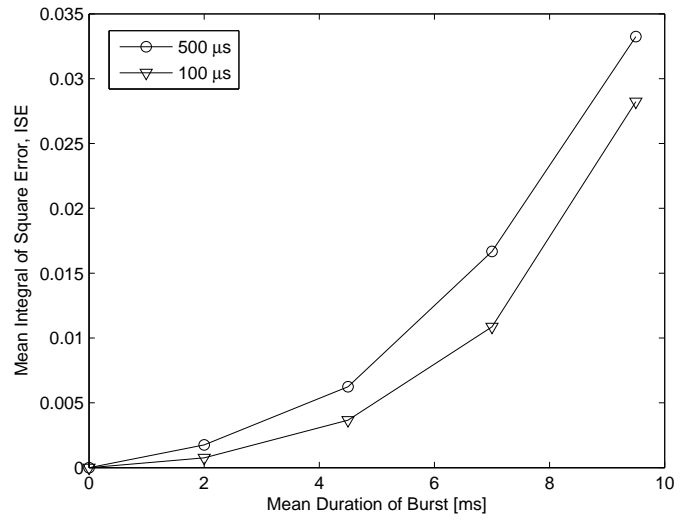

(b)

Figure 10: IAE vs Mean Error Burst Duration (a) and ISE vs Mean Error Burst Duration (b).

CNs representing the electrical drives is immediately detected and notified to the application layer of the protocol which, consequently, logs it in the Error History Object (a data structure recipient of all the errors occurring during network operation). An analogous procedure is undertaken by EtherCAT. Nonetheless, the electrical drive is not updated anymore, possibly causing serious drawbacks to the equipment it controls. Hence, in order to withstand the occurrence of (possibly permanent) physical layer errors, the drives have firstly to be able to detect the errors and then they need to implement suitable recovery procedures. As a possible technique of error detection, a drive could maintain a counter which is incremented each time a new set-point value is written by the RTE network on the input buffer of the drive itself. If during operation the drive gets several times the same set-point value from its input buffer, it checks the counter: if this latter has been correctly incremented, then it means that the set-point values have been actually received and that, in this case, they all have the same value. Conversely, if the counter has not been incremented, then the set-point values have not been received meaning that an error has occurred. 


\section{Conclusions}

RTE networks are powerful communication systems specifically designed to provide deterministic as well as hard real-time data transfer in modern factory automation systems. As such, they are particularly suitable for motion control applications and it is likely that, in the near future, they will be even more employed in these contexts.

In this paper we took into consideration two well known RTE networks, Ethernet POWERLINK and EtherCAT, and we analyzed their employment in a two-axis coordinated motion control application represented by the tracking of a circular trajectory. In particular, the networks were used to deliver the velocity set-points to the electrical drives that control the axis.

For both the networks we provided a theoretical analysis that allowed to evaluate some of their most relevant performance indexes. Then we presented the outcomes of a set of numerical simulations carried out in order to characterize the behavior of the coordinated motion control application. In particular, we simulated operational scenarios characterized by the presence of some types of errors, typically deriving from the employment of communication networks, especially in industrial environments. The obtained results showed the suitability of both the networks for the application as well as their ability to cope with anomalous error conditions.

Some interesting future developments may be envisaged from the analysis carried out in this paper. Indeed, as a natural prosecution of the work, the proposed configurations could be actually implemented and the results obtained from practical experiments compared with those shown in this work, giving the possibility of tuning the simulation models. Another appealing research direction is likely represented by the direct implementation of the velocity control loops on the controller resulting in a complete Networked Control System (NCS) [22]. Although such a solution is technically viable, and explicitly mentioned in [2], it is still not yet widely adopted (an example, however, is provided in [42] but for a system with non critical transmission times). The benefits deriving from such a new architecture would be relevant. For example, the firmware of the electrical drives results simpler; moreover, the implementation of the control algorithms, which in this case takes place on the controller, would benefit of the software tools typically available for such powerful devices (high level programming languages, compilers, debuggers, diagnostic procedures, etc.) which could significantly help (and speed up) the developing phase. Finally, the centralization of the control loops 
might allow for a more effective coordination of the electrical drives.

\section{References}

[1] T. J. Williams, A reference model for computer integrated manufacturing: a description from the viewpoint of industrial automation, Instrument Society of America, 1989.

[2] IEC 61800-7: Adjustable speed electrical power drive systems - part 7: Generic interface and use of profiles for power drive systems (November 2007).

[3] IEC 62390 technical report: Common automation device - profile guideline (January 2005).

[4] T. Sauter, The continuing evolution of integration in factory automation, IEEE Industrial Electronic Magazine 1 (1) (2007) 10-19.

[5] Industrial communication systems, in: R. Zurawski (Ed.), The Industrial Information Technology Handbook, CRC Press, 2005, pp. 37.147.16 .

[6] J. P. Thomesse, Fieldbus technologies in industrial automation, Proceedings of the IEEE 93 (6) (2005) 1073-1101.

[7] IEC 61158-5: Digital data communications for measurement and control - fieldbus for use in industrial control systems - parts 5 and 6: Application layer service definition and protocol specification, communication model type 3 (January 2000).

[8] EN 50325-2: Industrial communication subsystem based on ISO 11898 (CAN) for controller-device interface -part 2: DeviceNet (June 2000).

[9] ControlNet International, ControlNet specifications (March 1998). URL www.odva.org

[10] CAN In Automation, International Users and Manufacturers Group e.V., CANopen Application Layer and Communication Profile, CiA/DS 301, Version 4.01 (June 2000).

[11] F. Benzi, G. Buja, M. Felser, Communication architectures for electrical drives, IEEE Trans. on Industrial Informatics 1 (1) (2005) 47-53. 
[12] M. Felser, Real-time Ethernet-Industry prospective, Proceedings of the IEEE 93 (6) (2005) 1118-1129.

[13] Sercos International, Sercos Specifications (November 2001). URL www.sercos.org

[14] J. R. Moyne, D. M. Tilbury, The emergence of industrial contol networks for manufacturing control diagnostics and safety data, Proceedings of the IEEE 95 (1) (2007) 29-47.

[15] J. D. Decotignie, Ethernet-based real-time and industrial communications, Proceedings of the IEEE 93 (6) (2005) 1102-1117.

[16] IEEE 802.3 standard: Carrier sense multiple access with collision detection (CSMA/CD) access method and physical layer specifications (October 2000).

[17] G. Buttazzo, Hard Real-Time Computing Systems, Springer, 2005.

[18] IEC 61158: Digital data communications for measurement and control - fieldbus for use in industrial control systems - parts 2 to 6 (January 2000).

[19] IEC 61784: Digital data communications for measurement and control - part 2: Additional profiles for ISO/IEC 8802-3 based communication networks in real-time applications (November 2007).

[20] Ethernet POWERLINK Standardization Group, Ethernet POWERLINK Communication Profile Specification V. 2.0 (2003).

URL http://www. ethernet-powerlink.org

[21] EtherCAT Technology Group, EtherCAT: Ethernet for Control Automation Technology (2003).

URL http://www. ethercat.org

[22] J. P. Espanha, P. Naghshtabrizi, Y. Xu, A survey of recent results in networked control systems, Proceedings of the IEEE 95 (1) (2007) 138162.

[23] T. Li, Y. Fujimoto, Control System with High-Speed and Real-Time Communication Links, IEEE Trans. on Ind. Electr. 55 (4) (2008) 15481557. 
[24] S. Soucek, T. Sauter, Quality of Service Concerns in IP-Based Control Systems, IEEE Trans. on Ind. Electr. 51 (6) (2004) 1249-1258.

[25] W. J. Kim, K. Ji, A. Ambike, Real-Time Operating Environment for Networked Control Systems, IEEE Trans. on Automation Science and Engineering 3 (3) (2006) 287-296.

[26] F. L. Lian, J. K. Yook, D. M. Tilbury, J. Moyne, Network Architecture and Communication Modules for Guaranteeing Acceptable Control and Communication Performance for Networked Multi-Agent Systems, IEEE Trans. on Industrial Informatics 2 (1) (2006) 12-24.

[27] P. C. Richardson, W. Xiang, S. Mohammad, Performance Analysis of a Real-Time Control Network Test Bed in a Linux-Based System with Sporadic Message Arrival, IEEE Trans. on Industrial Informatics 2 (4) (2006) 231-241.

[28] A. Willig, A. Wolisz, Ring stability of the profibus token-passing protocol over error-prone links, IEEE Trans. on Ind. Electr. 48 (5) (2001) $1025-1033$.

[29] G. Cena, L. Seno, A. Valenzano, S. Vitturi, Performance analysis of ethernet powerlink networks for distributed control and automation systems, Computer Standards and Interfaces 31 (3) (2009) 566-572.

[30] IEC 61158-3,4: Digital data communications for measurement and control - fieldbus for use in industrial control systems - parts 3 and 4: Application layer service definition and protocol specification, communication model type 8 (January 2000).

[31] J. Jasperneite, M. Schumaker, K. Weber, Limits of increasing the performance of industrial Ethernet protocols, in: Proc. of ETFA 2007, Patras, Greece, 2007.

[32] G. Prytz, A performance analysis of EtherCAT and PROFINET IRT, in: Proc. of IEEE ETFA, Hamburg, Germany, 2008, pp. 408-415.

[33] OPNET Technologies, Opnet modeler user manual. URL http://www.opnet.com 
[34] MathWorks, Simulink.

URL http://www. mathworks.com

[35] K. C. Lee, S. Lee, M. H. Lee, Worst case communication delay of realtime industrial switched ethernet with multiple levels, IEEE Trans. on Ind. Electr. 53 (5) (2006) 1669-1676.

[36] V. M. Sempere, J. Silvestre, Multimedia Applications in Industrial Networks: Integration of Image Processing in Profibus, IEEE Trans. on Ind. Electr. 50 (3) (2003) 440-449.

[37] M. A. Duarte-Mermoud, R. A. Prieto, Performance index for quality response of dynamical systems, ISA Transactions 43 (1) (2004) 133-151.

[38] N. Navet, Y.-Q. Song, F. Simonot, Worst-case deadline failure probability in real-time applications distributed over controller area network, Journal of System Architecture 46 (7) (2000) 607-617.

[39] J. Broster, A. Burns, G. Rodriguez-Navas, Comparing real-time communication under electromagnetic interference, in: Proc. of 16th Euromicro Conference on Real-Time Systems, Catania, Italy, 2004, pp. 45-52.

[40] E. N. Gilbert, Capacity of a burst-noise channel, The Bell System Technical Journal 39 (1960) 1253-1265.

[41] European Committee for Electrotechnical Standardization, Electromagnetic compatibility (EMC) - Part 4-4: Testing and measurement techniques - Electrical fast transient/burst immunity test (April 2005).

[42] L. Yunhua, Y. Liman, Y. Guilling, Network-based coordinated motion control of large-scale transportation vehicles, IEEE/ASME Transactions on Mechatronics 12 (2) (2007) 208-215. 